\title{
THE ROLE OF SUPERSYMMETRY PHENOMENOLOGY IN PARTICLE PHYSICS
}

\author{
James D. Wells* \\ Physics Department, University of California, Davis, CA 95616 \\ Theory Group, Lawrence Berkeley National Lab, Berkeley, CA 94720
}

\begin{abstract}
Supersymmetry phenomenology is an important component of particle physics today. I provide a definition of supersymmetry phenomenology, outline the scope of its activity, and argue its legitimacy. This essay derives from a presentation given at the 2000 SLAC Summer Institute.
\end{abstract}

*Supported in part by the Department of Energy and the Alfred P. Sloan Foundation

(C) 2000 by James D. Wells. 


\section{Introduction}

The talk I delivered on "Supersymmetry Phenomenology" at the 2000 SLAC Summer Institute was a relatively standard talk on the definitions, motivations and research activities associated with supersymmetry phenomenology. Being the energetic conference that SSI is, much of the interesting discussion occurred after my talk. Participants asked me many challenging questions about supersymmetry and supersymmetry phenomenology. I enjoyed these exchanges and think they were at least as useful to me and the participants as was the talk. In this proceedings write-up, I would therefore like to emphasize the relevant post-talk issues as an addendum to the actual talk.

The purpose of this write-up is not to restate the meaning of supersymmetry and the basic moving parts of the theory. Some of this was covered in the talk, and much of it is covered admirably in pedagogical reviews of the subject. quickly and directly into my view of supersymmetry phenomenology.

We should start with a definition of "supersymmetry phenomenology" that is accurate, useful, and inclusive for all those who view themselves as working on the subject. Supersymmetry phenomenology attempts to answer four questions: (1) How can supersymmetry account for phenomena already measured and quantified? (2) How can supersymmetry resolve its own induced problems? (3) How can supersymmetry be found at future experiments? And, (4) how can supersymmetry be killed?

If we look at any one of those four elements of supersymmetry phenomenology in isolation, the field appears unmoored and speculation chasing. For example, if the field were interested only in question (3), the whole study would be without motivation. If the field were only concerned with question (1) practitioners would be charged with engaging in post-facto natural philosophy rather than scientific inquiry. The legitimacy of supersymmetry phenomenology derives from its commitment to address all questions raised above, and, just as important, the studies to date demonstrate very encouraging answers to those questions.

I would like to discuss, essay style, each of the questions in turn, and try to convince and remind the reader how much progress has been made answering them, and how much more work is still required. 


\section{How can supersymmetry account for the known?}

I would not say any known phenomena begs for supersymmetry, but a supersymmetric theory appears to make the natural world more understandable. For example, we know that the Higgs sector in the standard model has quadratic divergences and is likely unstable to non-trivial physics at high mass scales. Supersymmetry elegantly solves this instability problem by protecting hierarchies with a symmetry.2

Furthermore, the renormalization group evolution of Higgs masses in supersymmetry naturally induces electroweak symmetry breaking. This is often called "radiative electroweak symmetry breaking" because the radiative corrections (RGE evolution) propel the Higgs mass squared to negative values. And most interestingly, the minimal supersymmetric standard model, with a large top Yukawa coupling, generically predicts only one scalar mass squared going negative (up-Higgs), whereas all the remaining scalar masses (Squarks, sleptons, sneutrinos) remain positive. One can almost raise electroweak symmetry breaking to the level of a prediction of an $S U(3) \times S U(2) \times U(1)$ supersymmetric theory with our known particle content and Yukawa couplings. This is perhaps too strong, but one should realize that radiative electroweak symmetry breaking was discovered and understood 3 well after supersymmetry was introduced as a possible component to nature. As we will see in several other examples, this pattern of finding good things flowing from a supersymmetry hypothesis is encouraging.

Another example of supersymmetry's success is the explanation for the ratios of gauge couplings and Yukawa couplings we measure. Gauge coupling unification and Yukawa unifications are based on model dependent assumptions extending beyond the simplest supersymmetrizing of the standard model. Namely, they imply unification either Grand Unifications (GUTs) or some type of string unification. Yukawa unification, and the predictions derived from that are highly sensitive to matter content at the high scale. For this reason I will not attempt to force my positive opinions about this on others. However, I think the success of gauge coupling unification is extraordinary and wish to make a few comments on this.

Most of us have seen ad nauseum the graphs of the three gauge couplings unifying at a high scale in supersymmetry but not in the standard model. I think this suggests supersymmetry is the right way to go. Of course, it could be an accident that all three gauge couplings crossed at the same place in supersymmetry. Let's for a moment as- 
sume that there is nothing significant about that. Observers must also witness another accident: the three gauge coupling RGE trajectories cross at a scale that is not too low to be ruled out by proton decay experiments and cross not too high to be meaningless when combined with gravity. To me, this constitutes another interesting accident. Just like on the roadways, one accident is a curiosity, two accidents side-by-side is a real effect requiring deeper understanding of what happened.

It is true that the more accurate measurements of the last few years at LEP and SLC clearly show that extrapolation to high scales gives a small mismatch of couplings at the unification scale in a supersymmetric theory. That does not mean there is a problem with gauge coupling unification. It is almost certain that there are threshold corrections at the high-scale that will not enable one to simply take low-scale couplings and get exact unification at the high scale. There must be a slight mismatch. However, if the unification is perturbative, and the GUT group does not contain extraordinary amounts of matter, the mismatch should only be a few percent. Indeed, this is what is found.

The success of gauge coupling unification in a particular theory is measured by the three gauge couplings being "within specs" (within the variations inducible by threshold corrections) at the high scale. Supersymmetry easily passes this test, theories springing from the standard model do not.

\section{How can supersymmetry resolve its own problems?}

As with many things in life, an apparent solution to one problem can lead to even more problems. Buying a dog to protect you from burglars may only lead to your couch being chewed up. Whenever you introduce a new theory (supersymmetry) to supplant an old theory (standard model), you run the risk of spoiling all the successful predictions and explanations that we've held dear for so long. For example, small flavor changing neutral currents (FCNC) seen by experiments are mostly natural within the standard model. Supersymmetric descriptions, on the surface, have no preference for small FCNC effects over large effects. This is a potential problem.

However, the resolution to many of these questions is wrapped up in supersymmetry breaking. Some supersymmetry breaking mechanisms, such as gauge mediated

supersymmetry breaking, automatically solve many of these types of problems. 1 Some 
theories do not automatically, or naturally, solve the problems. What we must keep in mind is that supersymmetry is not one theory — it's a multitude of related theories (with only some non-negotiable properties in common) that each have strengths and weaknesses in describing what we already know. The supersymmetric theories that only have weaknesses get ignored over time.

As one illustration of how supersymmetry phenomenology studies have better understood how internal inconsistencies can be resolve, we turn to proton stability. In the standard model, lepton number conservation and baryon number conservation is an accidental global symmetry at the renormalizable level. For this reason, the proton is remarkably stable within the standard model framework.

In supersymmetry, as is the case in most beyond-the-standard model theories, proton stability is not even approximately automatic. The most general gauge invariant, Lorentz invariant lagrangian does not respect baryon and lepton number conservation. Somehow they must be banished. We all have a sense of what it means to solve a problem, and part of that solution is not to add epicycles to a theory that is apparently breaking down. However, supersymmetry phenomenologists have identified several elegant explanations for proton stability. One such explanation is R-parity.

R-parity is well-motivated in that it is a simple $Z_{2}$ symmetry that has no discrete gauge anomalies, and so could be derivable from a gauge symmetry. tant for our confidence that the discrete symmetry has a solid underpinning, and won't be violated by quantum gravity effects. Furthermore, the requisite continuous gauge symmetry is $U(1)_{B-L}$ which is contained in many of our formulations of grand unified theories. We can see R-parity as coexisting nicely with grand unified theories, gauge coupling unification, and proton stability, all within the same theoretical framework. For these reasons, R-parity is not an ad hoc assumption in my view.

The introduction of R-parity has another important, non-trivial implication: the lightest supersymmetric particle is stable. Of course, stable particles can be cosmological relics, and the first discussion of stable LSP relics exclaimed relief that it was not a cosmological disaster. 0 Quickly after that, it became apparent that not only is the LSP allowed by cosmology, but it might be preferred since its interaction strength with other particles can be just right to provide a good cold dark matter candidate.

I would say that the above story parallels the successful beginning history of many 
good ideas. The LSP was not dreamed up to solve the cold dark matter problem like other candidate particles were, but rather fell out of a self-consistent complete picture of the supersymmetric theory when attempting to solve its own consistency issues. And keep in mind, dark matter is a real observational and experimental problem that does not seem to be explainable within the standard model. Nevertheless, it remains to be seen if the LSP is truly the dark matter.

\section{How can supersymmetry be discovered?}

Post-facto explanations for electroweak symmetry breaking, Higgs sector quantum stability, Yukawa coupling ratios, and gauge coupling unification are encouraging, but they are not the end of the story. Additional phenomena confirming the explanations are required. Supersymmetry in fact has many unique and discerning signatures at high energy colliders, and elsewhere, to solidify its claims on nature. This is in stark contrast to many speculative theories that have no correlating phenomena and follow the "one observation, one explanation" pattern.

I will not attempt to go into any detail how supersymmetry could be found at highenergy colliders. My approach here is somewhat paradoxical because more studies and more solid analysis have been plied to this question than any other, in my estimation.

I simply refer the reader to the many excellent reviews on the subject. $⿴$ These studies show that careful predictions and anticipations combined with careful experimental preparation and analysis are all needed to find supersymmetry and accurately interpret new signals. Theorists and experimentalists engaged in any of these aspects contribute substantially to the resolution of the question posed for this section.

We should not forget that many smaller scale experiments, such as electric dipole moment measurements and $g-2$ measurements, contribute to our understanding of the natural world, and by implication the allowed form of a valid supersymmetric theory. Another important non-collider experiment includes the search for dark matter. These searches take on many guises, including cryogenic table top experiments, annihilations into photons in the galactic halo, and antiproton searches in the galactic halo. The prediction of many supersymmetric models is that evidence of LSP scattering would appear when the experiments get an order of magnitude or two more sensitive. 


\section{How can supersymmetry be killed?}

A common concern for any new theory is how it can be killed, or falsified. This is a complex issue that must combine technological capabilities (accelerators) with theoretical prejudices (what scale is supersymmetry?). This question, in my view, has value only inasmuch as it forces the respondent to formulate more detailed questions on the theory. For example, an easier subsidiary question would be "How can we rule out the bino LSP as an explanation for the dark matter?" or "How can we rule out supersymmetry as having an important role in electroweak symmetry breaking?" or "How can we determine if apparent unification of gauge couplings in supersymmetry is indeed just an accident?" These questions are still hard but they are easier breakdowns of the looming question: how do we determine if all of this is bunk?

These questions necessarily take on a negative tone, and are not pleasant to confront for a supersymmetry enthusiast. An unacceptable answer, but a technically correct answer, would be "we will never know." As scientists, we must make value judgements on how best to lasso natural law and try our best to figure it all out. Quixotic pursuits of nonviable theories run counter to these goals.

The community has set several standards on answering these questions. One is the careful evaluation of supersymmetric parameter space measured against a naturalness or finetuning criteria. For example, if supersymmetry governs electroweak symmetry breaking, the couplings and masses, it is argued, must be within reasonable bounds. Numerous papers have studied these questions in detail 9 Although they are oftened phrased positively ("We should see supersymmetry at future collider X"), they set the tone for when people should give up on supersymmetry. Let's call this the "relevance standard," because if supersymmetry parameters are outside the specified range for relevance to a problem, it is no longer motivated.

We are currently far from straying outside the "relevance standard", as judged by criteria set forth in the current literature and the criteria implied in the original works on

supersymmetry phenomenology 10 If the parameter space of relevant supersymmetry were mapped as a long drive between San Francisco and New York City, our current experiments have taken us only about as far as lowly Elko, Nevada.

Note also, it is vitally important to the integrity of the "relevance standard" that 
question (3) is answered in utter completeness, since we continue to find forms of supersymmetry that are extremely difficult to discover at colliders. For example, in many forms of anomaly mediated supersymmetry breaking, the lighter sparticles are nearly degenerate, and the lightest sparticle is invisible to the detectors. 11 Therefore, all that is produced in high energy collisions at, say, the LHC are a couple of very soft pions swamped by enormous backgrounds that cannot be overcome. Partial progress has been made on this issue, but other difficulties have been recognized and there are surely more discovery subtleties that we have not yet contemplated.

An even stricter application of the "relevance standard" can be found in considering the question, "What experiments must be performed to rule out supersymmetric theories that produce a bino with cosmologically significant cold dark matter density?" Depending on the precise nature of the theory, very detailed answers can be given and predictions on the success of colliders in finding such theories can be promulgated.12

There is one other standard, which I will call the "technology standard." This is a very practical standard, where you might hear it being applied when someone says, "If the LHC and NLC do not find supersymmetry I will give up on supersymmetry." On the surface it sounds silly, because nature does not care if the accelerator guy can only squeeze $7 \mathrm{TeV}$ out of the beams. But without additional planned accelerators in the lifetime of that physicist, there may not be need to address the prospects of supersymmetry manifesting itself at higher energies. All questions related to supersymmetry become moot to such a hardline phenomenologist. I do not like this standard for many reasons, some of which are implicit above. The main argument against it is that each generation applying it puts an arbitrary divider line on theory viability based on what they think the last machine, or last probing experiment can be accomplished in their professional lifetime. There is room to motivate and encourage better technology and better ideas, and supersymmetry might be a part of that argument in the future, or it might not. But the argument will always need to be made.

\section{Conclusion}

There remain several more questions presented to me at the Institute meeting that I would like to address in the conclusion. Many people would like to know from super- 
symmetry phenomenologists what percentage chance we give supersymmetry of being right. I have no idea. Supersymmetry is not running for city council against other welldefined candidates. The only thing I would say is that it appears to be the most attractive next step in our quest to get a deeper understanding of the natural world. I could never feel confident in judging its potential success on a more absolute scale because I am certain that I have not imagined all possibilities.

The next most asked question at the Institute along these lines was "What if supersymmetry isn't right? Doesn't that mean a lot of people have really wasted their time?" One answer to this question is that supersymmetry phenomenologists who study how supersymmetry should show up at experiments in general, and high energy colliders in particular, play a major role in helping experiments. The helpful input spans the full range from detector design to better techniques for data analysis and signal extraction. Remember, these experiments are multi-million dollar machines, sometimes multi-billion dollar machines, and having a few theorists around who are actively helping in this regard can only be a good thing. It increases the chances of finding a new signal, whether it be supersymmetry or not, and it helps maximize the interpretative scope of the data already taken (e.g., are $\mu=0$ theories viable?). These skills and activities are then transferrable over a wide range of theory-experiment searches for new physics, whatever the underlying theory might be.

A more substantive reply to this question must quarrel with the premise. The subtle premise of the question is that all worthwhile activity must be guaranteed at the start to terminate in a usable product or textbook theory. A quick survey of the entire scientific endeavor, not just particle physics, demonstrates the folly of this attitude. For example, if you are attempting to find a drug that cures a disease, you do not require a guarantee of success before starting. You start your work with a thorough knowledge of all the past sputtered attempts and you make regular evaluations of your work to determine if your approach can still lead to success. You get even more encouraged, and the pharmaceutical company gives you raises, if it is clear that your approach appears to be not only viable but more promising than anyone elses'. In the end, some of us will find the drugs that work, some of us won't, and hopefully very few of us will kill our trial subjects. But progress is made as a community of researchers trying to answer well-posed questions from different angles.

Our field is the high-energy frontier, and our questions are "What causes elec- 
troweak symmetry breaking?", "What is the dark matter?", "Why are the neutrinos so light?", "What causes CP violation?", and "What's next on the horizon?" Although many of us work in different aspects of supersymmetry phenomenology because we think it is promising, the experiments are what keep us tethered.

We are wed to the questions, not the hypothesized answers.

\section{References}

[1] The talk is meant to be readable and can be found at http://walden.ucdavis.edu/jwells/archive/ssi00.ps.

[2] For example, S. P. Martin, “A supersymmetry primer,” hep-ph/9709356.

[3] L. Ibanez and G. G. Ross, Phys. Lett. B110, 215 (1982); L. Ibanez, Phys. Lett. B118, 73 (1982); J. Ellis, D. V. Nanopoulos and K. Tamvakis, Phys. Lett. B121, 123 (1983); L. Alvarez-Gaume, J. Polchinski and M. B. Wise, Nucl. Phys. B221, 495 (1983).

[4] G. F. Giudice and R. Rattazzi, Phys. Rept. 322, 419 (1999) [hep-ph/9801271].

[5] L. M. Krauss and F. Wilczek, Phys. Rev. Lett. 62, 1221 (1989); L. E. Ibanez and G. G. Ross, Phys. Lett. B260, 291 (1991); R. N. Mohapatra, Phys. Rev. D34, 3457 (1986); A. Font, L. E. Ibanez and F. Quevedo, Phys. Lett. B228, 79 (1989); S. P. Martin, Phys. Rev. D46, 2769 (1992) [hep-ph/9207218].

[6] S. Weinberg, Phys. Rev. Lett. 50, 387 (1983).

[7] H. Goldberg, Phys. Rev. Lett. 50, 1419 (1983).

[8] For example, M. Carena, R. L. Culbertson, S. Eno, H. J. Frisch and S. Mrenna, Rev. Mod. Phys. 71, 937 (1999) [hep-ex/9712022].

[9] J. Ellis, K. Enqvist, D. V. Nanopoulos and F. Zwirner, Mod. Phys. Lett. A1, 57 (1986); R. Barbieri and G. F. Giudice, Nucl. Phys. B306, 63 (1988); G. W. Anderson and D. J. Castano, Phys. Rev. D52, 1693 (1995) [hep-ph/9412322]; J. L. Feng, K. T. Matchev and T. Moroi, Phys. Rev. D61, 075005 (2000) hep-ph/9909334.

[10] For example, the Haber-Kane Physics Report on supersymmetry phenomenology from 1985 implied a "relevance standard" of superpartners being lighter than about $1 \mathrm{TeV}$, which is far from the current experimental limits: "We emphasize 
that the solution to the naturalness (fine-tuning) problem necessarily predicts that new physical phenomena must exist at a scale of $m_{w} / g \sim \mathcal{O}(1 \mathrm{TeV})$ or below. In the case of supersymmetry, this new physics consists of a spectrum of new supersymmetric particles (partners of the ordinary particles) which have masses no greater than about $1 \mathrm{TeV}$ and in some cases may be substantially lighter." H. Haber, G.L. Kane, Phys. Rep. 117, 75 (1985), quote from page 82.

[11] T. Gherghetta, G. F. Giudice and J. D. Wells, Nucl. Phys. B559, 27 (1999) [hepph/9904378]; J. L. Feng, T. Moroi, L. Randall, M. Strassler and S. Su, Phys. Rev. Lett. 83, 1731 (1999) [hep-ph/9904250]; J. F. Gunion and S. Mrenna, Phys. Rev. D62, 015002 (2000) [hep-ph/9906270].

[12] For example, J. Ellis, G. Ganis and K. A. Olive, Phys. Lett. B474, 314 (2000) [hep-ph/9912324]. 